\title{
A Technique to Suppress Speckle in Ultrasound Images using Nonlocal Mean and Cellular Automata
}

\author{
Monika Pathak ${ }^{1}$, Harsh Sadawarti ${ }^{2}$ and Sukhdev Singh ${ }^{3}$ \\ II. K. Gujral Punjab Technical University, Jalandhar, Punjab, India \\ 2Department of Computer Science, RIMT, Mandi Gobindgarh, Punjab, India \\ 3Multani Mal Modi College, Patiala, Punjab, India; tomrdev@gmail.com m
}

\begin{abstract}
Kidney ultrasound imaging is an economical, non invasive, real time diagnosis system which is used to measure abnormalities in the shape, size and location of the kidneys in the body. The presence of speckle noise degrades the visual quality of ultrasound images. The primary objective of the research under taken is to detect the renal stone in ultrasound images. The present research paper discusses an algorithm based on cellular automata and nonlocal mean for suppression of the speckle noise. The cellular automatons are dynamic systems to represent the particular problem in terms of specific pattern. This concept is exploited to distinguish the noise from the object being target. The kidney ultrasound images are denoised to detect presence of renal stone in the image. The concept behind the nonlocal mean is an assumption that the pixel being considered for denosing has strong connection with the surrounding area rather than the exactly nearly by pixels. The MLNC (Maximum Likelihood Neighborhood Computation) rules are applied to find the nearest value in the neighbour. The BEP (Border, Edge Preservation) rules are used to safeguard the edges between the regions. The samples of 55 clinical images of ultrasound are used for statistical analysis which is carried out by Signal to Noise Ratio (SNR), Mean Square Error (MSE), Peak Signal to Noise Ratio (PSNR). The result analysis shows MSE $=216.789$, SNR $=49.1569$ and PSNR = 68.022. The algorithm not only enhances the visual quality but also suppress unwanted speckle noise. The algorithm is dynamic in nature and can be used to de-noise SAR images, MRI and X-rays image. The method is adaptive in nature as cellular modeling can model low noise level.
\end{abstract}

Keywords: Adaptive Filters, Cellular Automata, Despeckling, Moor Neighborhood, Nonlocal Mean

\section{Introduction}

The ultrasound imaging is used for producing real time images of soft tissues in organs like kidney, uterus, heart, brain, liver, etc. It plays a major role in making medical imaging more popular due to its non-invasive nature, cost effective, real time imaging, and no harm to the human organ. In spite of popularity and usefulness, the ultrasound images suffer from a number of noises such as equipment image acquisition noise, environmental ambient noise and speckle noise due to unformatted reflection during scanning. The most prominent noise is the speckle noise which not only reduces the sharpness of the image but also hinders the diagnosis process. The source of speckle in the image is destructive coherent interferences of back scattered echoes from smaller objects in the ultrasound scanning ${ }^{1}$. The speckle pattern is a form of multiplicative noise and available everywhere in the ultrasound images. It not only degrades the visual quality but also makes it difficult to identify the presence of edges, boundaries and small objects like stone in the image.

The speckle needs to be suppressed to enhance the visual quality of the images. The early approaches for speckle suppression are the low-pass filters, wavelet transform, nonlinear and cellular automata based filters, etc. The low pass filters like homomorphic Wiener filters can effectively

${ }^{*}$ Author for correspondence 
suppress noise, but fail to preserve many useful fine details. On the same line of action the techniques like median filter and homomorphic filter were developed by Lee filter ${ }^{2}$, Kuan filter, Frost filter to suppress noise. In the next phase, concept of wavelet transform rapidly emerged. Initially, it was used for denosing single dimensional signal but later on it was modified to an adaptive wavelet threshold ${ }^{3}$ wherein combined approaches of a linear/ non-linear wavelet, transform wavelet and linear inverse wavelet transform were used to reduce noise on ultrasonic cardiogram images. Efros and Leung ${ }^{4}$ introduced the idea of self-similarity for texture synthesis and which was later on used in nonlocal mean noise filters. Wang et al. ${ }^{5}$ have developed methods based on nonlocal total variation to remove speckle noise.

In spite of such an extensive research work on suppressing speckle, these methods more or less are task specific and need to manage tradeoff between suppression of noise and preserving fine detail intermixed with the speckle.

The problem of speckle in ultrasound is inherited and unavoidable. The suppression of the speckle in the image is a traditional and a challenging task. The literature survey reveals various methods used to suppress the noise and enhance the visibility. In ${ }^{6}$ introduced denoising technique for ultrasound images to find the cancer region or real boundary of tumor region. The method used the cellular network to decide a template to find boundaries of the regions. The result analysis have shown significant enhancement of lesion boundary in the images. $\mathrm{In}^{7}$ developed a cellular automaton to extract the location information of follicles in the ultrasound image. The result analysis has shown successful detection of all large follicles.

In $^{8}$ presented a technique for speckle reduction of ultrasound image. The algorithm was implemented using EDK on ML310 embedded development platform. It is based on the aggressive region growing filter which selects a fixed size window region from input image around the central pixel. The algorithm starts with seed region and finds the homogeneity within the region. The homogeneity threshold value is compared with region homogeneity value and if it lies within the region then region grows otherwise different directions are explored to find homogeneity regions. The trimmed arithmetic mean filter and geometric mean filtering are applied on the largest window and the smaller respectively. The result shows successful speckle filtering within homogeneous regions and makes the edges sharper.

$\mathrm{In}^{9}$ proposed an algorithm for detection and identification of renal stones in ultrasound images. The features extraction and classification were performed using image features contrast and target shape features. The feature extraction algorithm was applied on sample images of stone and statistical properties were studied to identify the stone in the image. In a similar study ${ }^{10}$ the different features of ultrasound images were extracted which were based on intensity histogram and gray level co-occurrence matrix. The five intensity histogram features and nineteen Gray Level Co-occurrence Matrix (GLCM) features were used to classify the object in the images.

In $^{11}$ derived several multi-scale nonlinear threshold methods for the removal of speckle from ultrasound images. The algorithm used Gaussian and Laplacian local mixture distribution for each noise free wavelet coefficient.

$\mathrm{In}^{12}$ presented cellular automata for image noising, particularly for impulse noise. The cellular automata rules were constructed to filter impulse noise both from binary and gray scale images. The performance of the filter was compared with other classical median filters. The statistical analysis using PSNR has shown that the filter based on cellular automata gave better noise suppression over the classical methods. Subsequently Qadir et al. ${ }^{13}$ used and optimized the concept of the cellular automata to enhance performance. The concept of non-uniform cellular automata rules was constructed to filter the noise from both ordinary images and medical images. The algorithm has shown significant improvements over the traditional filters and took lesser time to denoise the images.

The literature survey reveals that the most significant region of interest is to denoise the ultrasound images. Thus the current study is aimed to denoise the image for detection of calculi in the kidney. It becomes more significant to suppress the noise while preserving the fine detail. The proposed algorithm in the current study is an attempt to suppress the speckle using most popular and effective tools namely cellular automata and nonlocal mean. The cellular automata are capable to generate any complex to complex pattern whereas nonlocal mean can address effect of other region on the local region under study.

In this paper, we have proposed a method which is based on cellular automata and non linear local mean to suppress speckle in the kidney images. The cellular automata represent arrangement of cell in Moor neighborhood and nonlocal means are considered to denoise 
the central pixel. The statistical analysis and visual observation show significant enhancement in the visual quality of the images.

\section{Cellular Automata for Denoising}

The cellular automata are discrete dynamic systems where lattice (cells) are arranged in a specific pattern to model the problem under study and finite states of lattice are evolved on the basis of transition rules ${ }^{14}$. The state of the lattice depends on the behavioral characteristics of the neighboring cell near to the central cell and it changes over time.

Let us consider $\mathrm{L}$ be regular lattice, $\mathrm{S}$ be a finite set of states, $\mathrm{N}$ a finite set of neighborhood.

The transition function

$$
f: S^{n} \rightarrow S \text { Wehere } \forall C \in N, \forall r \in L
$$

The lattice geometry is highly dependent on the arrangement of the neighborhood cells. The Von Neumann and Moor neighborhood are two well known arrangements of neighborhood. The simplest examples of these two are shown in Figure 1.

As shown in the Figure 1, the neighborhood cells are defined as the eight cells surrounding the cell, known as a Moore neighborhood whereas the cells directly above, below, to the left and to the right of the cell, known as the von Neumann neighborhood. The state of the cell is computed by its state in the previous step of a surrounding neighborhood of cells and is updated in discrete manner with reference to the time.

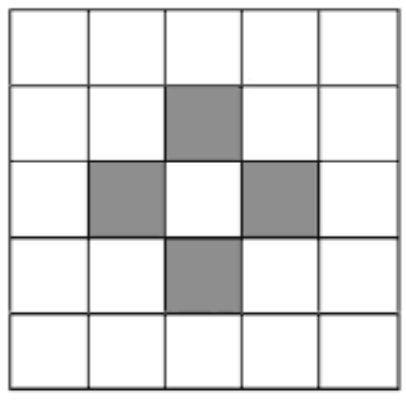

(a)

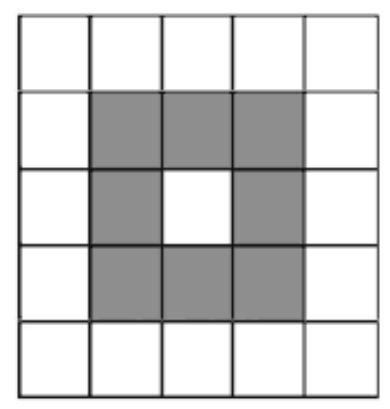

(b)
Figure 1. (a) The Von Neumann neighborhood, (b) The Moor neighborhood.
The construction of the cellular automata for speckle suppression is a two dimensional square lattice with Moor neighborhood ${ }^{15}$. The input image is covered to gray scale before using cellular automata and the image size is also optimized to reduce the computation overhead. The transitional rules are framed in pseudo code described in 4.1. The state of the cellular automata is a finite gray values ranging from $0-255$. The denoised value is the value of means derived from nonlocal mean computation. The concept behind the nonlocal mean is an assumption that the pixel being considered for denosing has strong connection with the surrounding area rather than the exactly surrounded pixels. The computation is based on local mean and nonlocal mean where nearest likelihood neighborhood rules are applied.

\section{Nonlocal Mean}

The denoising techniques usually use local structure for processing central pixel surrounded by a small set of pixels neighborhood. The Gaussian smoothing filter is one of the local technique used to denoise the pixel surrounded by local neighborhood. The fundamental assumption in local filtering is that the noise is oscillatory and image is piecewise smooth. The task of these filters is to remove the oscillatory pixels from piecewise smooth images. It has been observed that in most of the cases, oscillatory particles intermix with fine structured particles which results in loss of information. Sometimes local filtering results in blurring, stair casing and checkbox effects. The Gaussian Filtering ${ }^{16}$ which belongs to the class of local smoothing, which is based on assumption that gray values of an image are similar in the local spatial neighborhood. The limitation of this approach is non preservation of edges due to the locally similarly of gray values in the neighborhood. The frequency domain filtering ${ }^{17}$ is performed denoising over the frequency domain. On the other hand, the absence of high frequency results in small local variance of gray level values. Hence, frequency domain filters leads to outliers and artifacts at edges.

The nonlocal means (NL-means) model consists of denoising a gray level value at a point by averaging point values with similar structures (patches). Thus, not only the local gray level values of points are used to define similarity, but rather the values of a window around these points will also be considered.

An example of nonlocal mean is displayed in Figure 2, where $\mathrm{mo}, \mathrm{m} 1, \mathrm{~m} 2, \mathrm{~m} 3, \mathrm{~m} 4, \mathrm{~m} 5$ and $\mathrm{m} 6$ represent mean 
intensity of the rectangular regions respectively. The rectangular regions have their own mean values, as visual observation reveals that region with mean value $\mathrm{m}_{0}$ is closer to the regions having similar contrast and not the regions which are physically closer. But most of the time, the neighboring region have more impact and close association. The better approximation of the pixel is the nearest neighborhood. The vote to the nearest neighbour is key idea. For example, in Figure 2, the pixels enclosed in the region, where mean is $\mathrm{m}_{0}$ is better associated with region with values $\mathrm{m} 2$ and $\mathrm{m} 4$, as these region are closer to the region $\mathrm{m}_{0}$. Whereas $\mathrm{m} 3, \mathrm{~m} 5, \mathrm{~m} 6, \mathrm{~m} 1$ are far ways the region and have less impact on $\mathrm{m}_{0}$.

Let us suppose $\mathrm{P}_{0}, \mathrm{P}_{1}, \mathrm{P}_{2}$ represent different regions in the image. The Pixels $\mathrm{P}_{0}$ and $\mathrm{P}_{1}$ belong to similar neighborhood whereas pixels $\mathrm{p}_{0}$ and $\mathrm{P}_{2}$ have different neighborhood. Accordingly the nonlocal mean $\mathrm{P}_{1}$ have more influence on $\mathrm{P}_{0}$ as compare to $\mathrm{P}_{2}$. The pixel $\mathrm{P}_{0}$ will be denoised using no local mean with the following formula:

$$
N L(G)(p)=\sum_{p 2 \in v}^{v} \mathrm{~W}\left(p_{0}, \mathrm{p}_{2}\right) \mathrm{G}\left(\mathrm{p}_{2}\right)
$$

Where $\mathrm{G}$ is the noisy image, and weights $\mathrm{W}\left(\mathrm{P}_{0}, \mathrm{P}_{2}\right)$ a weighted average of each pixel.

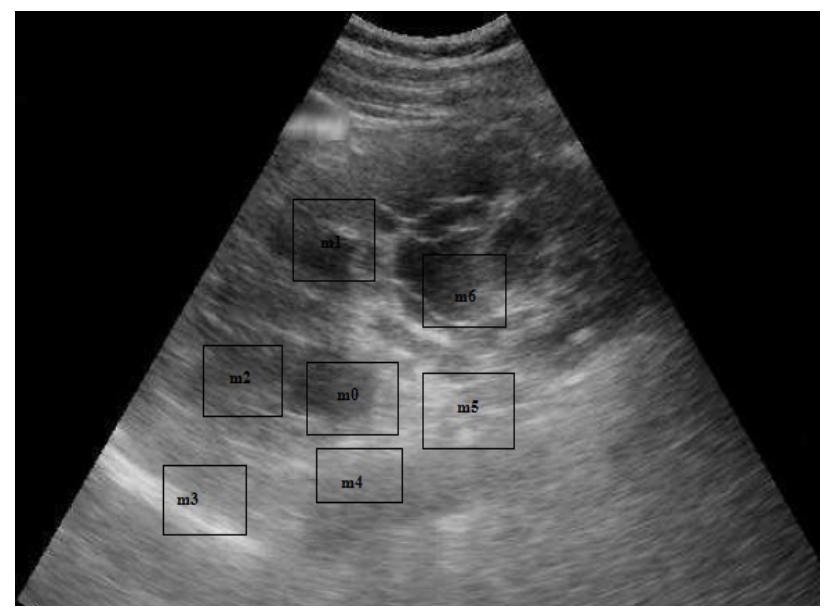

Figure 2. Demonstrates concept of nonlocal mean using self-similarity in an image.

\section{Algorithm}

The proposed algorithm is based on cellular automata which is defined by the Moor neighborhood and mean values of different neighboring regions are computed as $\mathrm{m} 0, \mathrm{~m} 1, \mathrm{~m} 2, \ldots$ .mn. The MLNC (Maximum Likelihood Neighborhood Computation) rules are applied to find the nearest value in the neighbour. The BEP (Border, Edge Preservation) rules are used to safeguard the edges between the regions.

The algorithm is as follows:

For each pixel position in noised image $(i, j)$

(i) Consider a Moor Neighborhood $(r=1)$ around pixel $(\mathrm{i}, \mathrm{j})$ to be denoised.

(ii) Consider cellular automata as shown in figure

(iii) In the cellular pattern, the Moor Neighborhood is considered around pixels positioned in neighboring locations with reference to the pixel $(i, j)$ to be denoised. The eight pixel locations with reference to pixel (i, j) are: (i-3r, j-3r), (i, j-3r), (i+3r, j-3r), (i-3r, j), $(i+3 r, j),(i-3 r, j+3 r),(i, j+3 r),(i+3 r, j+3 r)$

Where, $r$ is the radius of the Moor Neighborhood.

(iv) Compute gray mean value of pixels in the Moor Neighborhood around pixel $(\mathrm{i}, \mathrm{j})$ referred as $\mathrm{m}_{0}$.

(v) Subsequently, compute gray mean values of all the neighboring moor Neighborhood around pixel listed in step 2 and then named as $\mathrm{m} 1, \mathrm{~m} 2, \ldots \ldots \mathrm{m}_{\mathrm{n}}$, Where the value of $n$ depends on the value of $r$ i.e. value of the radius of Moor neighborhood.

(vi) Apply MLNC (Maximum Likelihood Neighborhood Computation):

- The pixel to be denoised is at $(\mathrm{i}, \mathrm{j})$ location with the gray scale value I and mean of gray scale value is $\mathrm{m} 0$.

- Compare $\mathrm{m}_{0}$ with $\mathrm{m}_{1}, \mathrm{~m}_{2}, \ldots . ., \mathrm{m}_{\mathrm{n}}$ and calculate difference in values as $d_{1}, d_{2}, \ldots . d_{n}$.

- Create a list (mList) of $\mathrm{m}$ values which are closer to the value of $m_{0}$, excluding extreme value if any in the group.

(vii) Apply BEP (Border, Edge Preservation):

- If all the neighborhood values are same, then there is a strong sigh of the presence of the border and pixel to be diagnosed may not belong to the neighbour group. The similar mean values are to be removed from mList.

- If some of the neighborhood values are similar or have a difference of one, then it may be an edge. Then pixel under consideration need not be affected by the neighborhood pixels.

- Find the average of mlist values and replace the average value with Pixel $(i, j)$ value.

The region considered for denoising around $\mathrm{m} 0$ is called local neighborhood whereas regions around $\mathrm{r} 1, \mathrm{r} 2, \ldots$ 


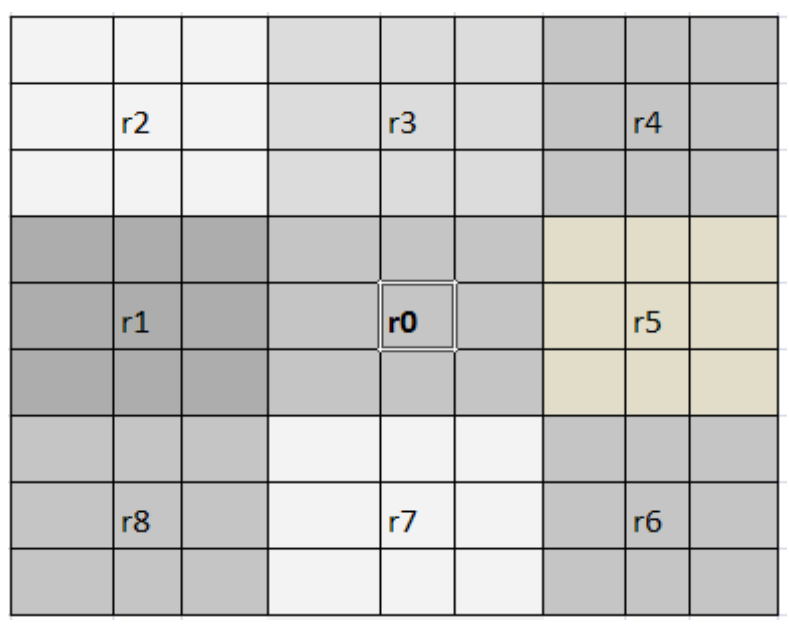

Figure 3. Local-neighborhood and surrounding neighborhood.

.,r6 are referred as surrounding regions. The central pixel in region $\mathrm{r} 0$ is denoised by considering mean gray values of local neighborhood $\mathrm{r} 0$ and surrounding neighborhood r1, r2, r3, r4, r5, r6, r7 and r8.

\section{Results and Conclusion}

The statistical analysis of the proposed algorithm is carried out on kidney ultrasound images. The following statistical parameters have been used for analysis:

\subsection{Signal to Noise Ratio (SNR)}

The SNR value is calculated using the formula:

$$
S N R=\frac{\mu}{\sigma}
$$

Where, $\mu$ is the mean calculated over the final image taken into consideration and $\sigma$ is the standard deviation of the images.

\subsection{Mean Square Error (MSE)}

The MSE measures change in denoised image after applies the denosing method on input image.

\section{可}

Where, $\mathrm{X}$ is an input image, $\mathrm{Y}$ represents denoised output image, $\mathrm{N}$ represent dimension of the image.

\subsection{Peak Signal to Noise Ratio (PSNR)}

The peak signal to noise ratio can be expressed as:

$$
P S N R=10 \log _{10} \frac{(255)^{2}}{M S E}
$$

Where, MSE is mean square error, and 255 represent maximum value of a pixel in the image.

The PSNR value represents closeness of the denoised image to the image before denoising. If the PSNR value is higher, then denoised image is closer to the original image.

The statistical analysis with statistical parameters is

\begin{tabular}{|c|c|c|c|}
\hline Noise Level & MSE & SNR & PSNR \\
\hline 0.01 & 216.789 & 49.1569 & 68.022 \\
\hline 0.02 & 357.172 & 44.6400 & 63.029 \\
\hline 0.03 & 500.039 & 40.7993 & 59.664 \\
\hline 0.04 & 639.312 & 38.4050 & 57.270 \\
\hline 0.05 & 777.474 & 36.3856 & 55.250 \\
\hline 0.06 & 915.958 & 34.7464 & 53.611 \\
\hline 0.07 & 1058.96 & 33.2956 & 52.160 \\
\hline 0.08 & 1184.92 & 32.1718 & 51.037 \\
\hline 0.09 & 1316.18 & 31.1212 & 49.986 \\
\hline
\end{tabular}
shown in the table below:

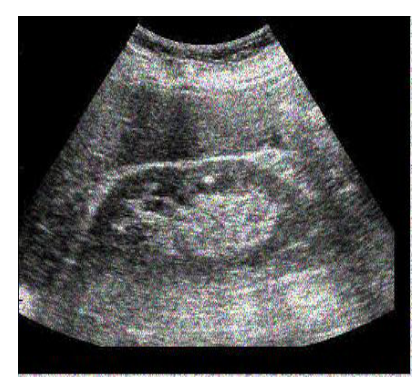

(a)

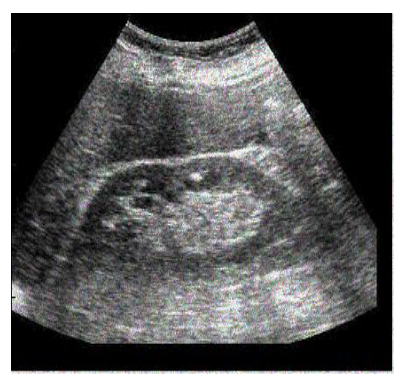

(c)

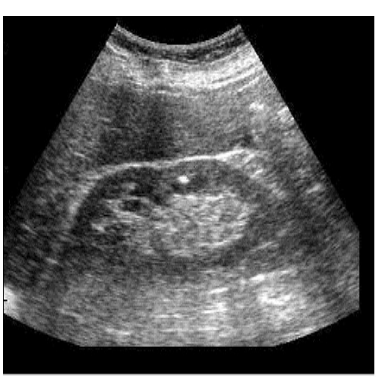

(b)

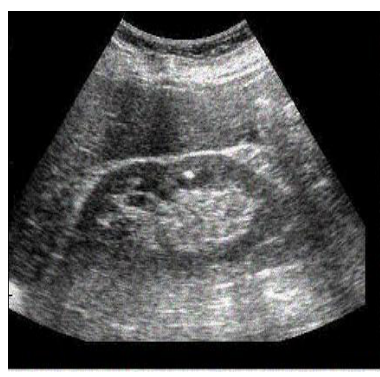

(d)
Figure 4. (a) and (b) are denosed image and (c) and (d) are denoised image after using proposed method.

The following graph shows relationship between PSNR and noise level. As the noise level increases PSNR values decrease. 


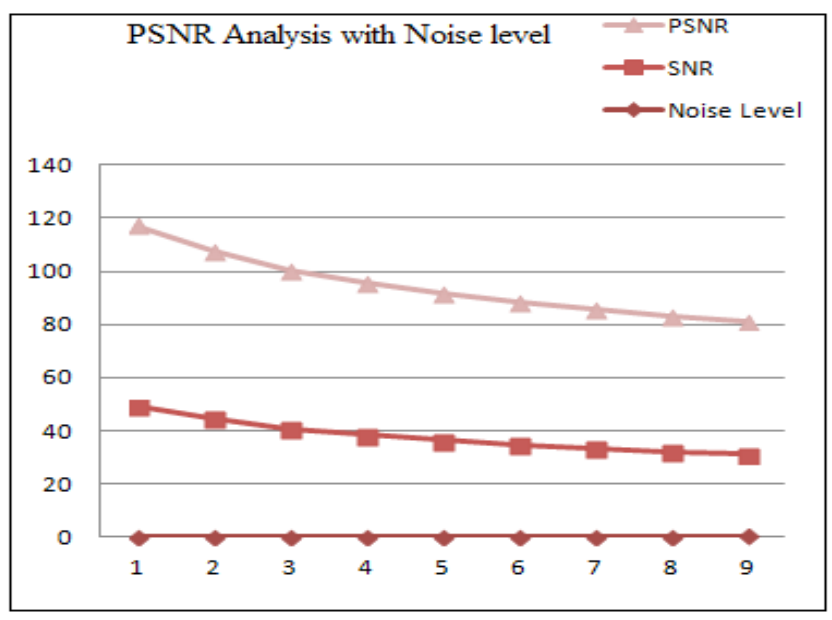

Figure 5. Shows progression of PSNR with noise level.

The greater the PSNR value better is the result. The statistical analysis shows significant enhancement in the ultrasound images. The samples of 55 clinical images of ultrasound are used for testing. This module will be extended for automatic detection of calculi in the ultrasound images. The performance of the proposed method can be enhanced by varying cellular automata transient rules. The current study has considered mean of local and surrounding areas for denoising the image. The statistical features of the local and surrounding areas like standard deviation and variance can also be exploited to find symmetric pattern in the ultrasound images.

\section{Acknowledgement}

Authors acknowledge the opportunity and support provided by I. K. Gujral Punjab Technical University, Jalandhar to conduct the present research work.

\section{References}

1. Loganayagi T, Kashwan KR. A Robust Edge Preserving Bilateral Filter for Ultrasound Kidney Image. Indian Journal of Science and Technology. 2015 Sep; 8(23).

2. Lee JS. Digital image enhancement and noise filtering by use of local statistics. IEEE Transactions on Pattern Analysis and Machine Intelligence. 1980; PAMI-2(2):165-8.

3. Chang SG, Yu B, Vetterli M. Adaptive wavelet thresholding for image denoising and compression. IEEE Transactions on Image Processing. 2000; 9(9):1532-46.

4. Efros A, Leung T. Texture synthesis by non parametric sampling. The Proceedings Seventh IEEE International conference on Computer Vision; Kerkyra. 1999. p. 1033-38.
5. Wang L, Xiao L, Huang L, Wei Z. Nonlocal total variation based speckle noise removal method for ultrasound image. 2011 4th IEEE International Congress on Image and Signal Processing (CISP); 2011 Oct. p. 709-13.

6. Park HK, Yoo BC, Ryu JG, Hwang KS, Nishimura T. The Speckle Reduced Ultrasound Images Using Cellular Neural Network with Effective Detection of Active Contour Model. Proceedings of the World Congress on Engineering and Computer Science; San Francisco. 2008. p. 1-5.

7. Viher B, Dobnikar A, Zazula D. Cellular Automata and Follicle Recognition Problem and Possibilities of using Cellular Automata for Image Recognition Purposes. International Journal of Medical Informatics. 1998; 49(2):231-41.

8. Mazumdar B, Mediratta A, Bhattacharyya J, Banerjee S. An Embedded System Design of Selective Window Speckle Noise Suppression Filter for Ultrasound Images. First International Conference on Industrial and Information Systems (ICIIS); Peradeniya. 2006. p. 324-7.

9. Tsao J, Chang LH, Lin C-H. Ultrasonic Renal-Stone Detection and Identification for Extracorporeal Lithotripsy. 27th Annual International Conference of the IEEE Engineering in Medicine and Biology; Shanghai, China. 2005. p. 6254-7.

10. Hafizah W M, Supriyanto E, Yunus J. Feature Extraction of Kidney Ultrasound Images based on Intensity Histogram and Gray Level Co-occurrence Matrix. Sixth Asia Modelling Symposium (AMS); Bali. 2012. p. 115-20.

11. Hossein R, Vafadust M, Abolmaesumi P. Speckle Noise Reduction of Medical Ultrasound Images in Complex Wavelet Domain Using Mixture Priors. IEEE Transactions on Biomedical Engineering. 2008; 55(9):2152-60.

12. Selvapeter JP, Hordijk W. Cellular Automata for Image Noise Filtering. World Congress on Nature and Biologically Inspired Computing (NaBic'09); Coimbatore. 2009. p. 193-7.

13. Qadir F, Peer MA, Khan KA. An Effective Image Noise Filtering Algorithm Using Cellular Automata. International Conference on Computer Communication and Informatics; Coimbatore. 2012. p. 1-5.

14. Rosin PL. Training cellular automata for image processing. IEEE Transactions on Image Processing. 2006; 15(7):2076-87.

15. Popovici A, Popovici D. Cellular automata in image processing. The 15th International Symposium on the Mathematical Theory of Networks and Systems. In: Gilliam DS, Rosenthal J, editors. Electronic Proceedings; 2002. p. 1-6.

16. Krissian K, Vosburgh K, Kikinis R. Westin C-F, Anisotropic diffusion of ultrasound constrained by speckle noise model. 2004. p. 1-12.

17. Maini R, Aggarwal H. A Novel Technique for Speckle Noise Reduction on Medical Images. International Journal of Applied Engineering Research. 2010; 5(1):1-8. 